age among all gynecological cancers. To examine the oncologic and reproductive outcomes of fertility-sparing surgery (FSS) compare to abdominal radical hysterectomy (ARH) in women with early stage CC.

Methods Retrospective data were analyzed from 121 patients with IA2-IB1 and IIA1 CC stages treated at NN Alexandrov National Cancer Centre of Belarus from 2009 to 2018.

Results A total of 83 patients met the FSS inclusion criteria. Thirteen patients were excluded. The rest of 70 patients were selected in FSS study (group 1). Patients were stratified for 3 types of FSS. The results of treatment in group 1 were compared with 51 patients (group 2), whom ARH was performed. Five-year overall survival and 5-year disease-free survival (DFS) were similar between the two groups $93.1 \% \quad(\mathrm{SE} \quad 4.0 \%)$ vs $98.0 \% \quad(\mathrm{SE} 2,0 \%), \mathrm{p}=0.431$; and $88,3 \%$ (SE 4.2\%) vs 92,1\% (SE 3.8\%), p=0.594, respectively. Similarly, 5-year DFS rate were comparable between groups for all the stages examined. During follow-up 9 pregnancies were achieved in 6 patients. Most pregnancies $(6 / 9,66.7 \%)$ and all deliveries (4) occurred in the ultraminimal FSS subgroup whose patients underwent amputation and pelvic lymphadenectomy.

Conclusions Within this population of early CC patients, equivalent oncologic outcomes have been achieved for FSS group were ultraminimal and minimally invasive approaches were used to compare with ARH group. The fertility-preserving procedure had clear advantages of less invasive access surgery in terms of reproductive outcomes compared to ART.

\section{EPV149/\#132 PERFORMANCE CHARACTERISTICS OF BRIEF FAMILY HISTORY QUESTIONNAIRE TO SCREEN FOR LYNCH SYNDROME IN WOMEN WITH NEWLY DIAGNOSED OVARIAN CANCERS}

${ }^{1} \mathrm{RS} \mathrm{Kim}^{*},{ }^{1} \mathrm{~A}$ Tone, ${ }^{2} \mathrm{R}$ Kim, ${ }^{3} \mathrm{M}$ Cesari, ${ }^{4} \mathrm{~L}$ Eiriksson, ${ }^{5} \mathrm{~T}$ Hart, ${ }^{6} \mathrm{~A}$ Lytwyn, ${ }^{7} \mathrm{M}$ Maganti, ${ }^{1} \mathrm{M}$ Bernardini, ${ }^{2} \mathrm{~A}$ Oza, ${ }^{3} \mathrm{~B}$ Djordjevic, ${ }^{3} \mathrm{~J}$ Lerner-Ellis, ${ }^{1} \mathrm{E}$ Van De Laar, ${ }^{8} \mathrm{D}$ Vicus, ${ }^{3} \mathrm{~A}$ Pollett, ${ }^{1} S$ Ferguson. ${ }^{1}$ Princess Margaret Cancer Centre/University of Health Network/Sinai Health Systems, Gynecologic Oncology, Toronto, Canada; ${ }^{2}$ Princess Margaret Cancer Centre/ University of Health Network/Sinai Health Systems, Medical Oncology and Hematology, Toronto, Canada; ${ }^{3}$ University of Toronto, Laboratory Medicine and Pathobiology, Toronto, Canada; ${ }^{4}$ Juravinski Cancer Centre, McMaster University, Gynecologic Oncology, Hamilton, Canada; ${ }^{5}$ Ryerson University, Psychology, Toronto, Canada; ${ }^{6}$ McMaster University, Pathology and Molecular Medicine, Hamilton, Canada; ${ }^{7}$ Princess Margaret Cancer Centre, Biostatistics, Toronto, Canada; ${ }^{8}$ Sunnybrook Health Sciences Centre, Gynecologic Oncology, Toronto, Canada

\subsection{6/ijgc-2021-IGCS.219}

Objectives Ovarian cancer (OC) is the third most common Lynch syndrome (LS)-associated cancer in women but there is no established screening strategy to identify LS in this population. We have previously validated the 4-item brief Family History Questionnaire (bFHQ) in endometrial cancers. The objective of this study was to assess whether bFHQ can be used as a screening tool to identify women with OC at risk of LS.

Methods In this multicenter prospective cohort study, women with OC completed bHFQ, extended Family History Questionnaire (eFHQ; encompassing Amsterdam II criteria, Society of Gynecologic Oncology 20-25\% criteria and Ontario Ministry of Health criteria), immunohistochemistry (IHC) for mismatch repair (MMR) proteins and universal germline testing for LS. Performance characteristics were compared between bFHQ, eFHQ, and IHC.
Results of 215 participants, 169 (79\%) were evaluable with both bFHQ and germline mutation status; 12 of these 169 were confirmed to have LS (7\%). Nine of 12 patients $(75 \%)$ with LS were correctly identified by bFHQ, compared to 6 of $11(55 \%)$ by eFHQ and 11 of $13(85 \%)$ by IHC. The sensitivity, specificity, positive predictive values and negative predictive values of bFHQ were 75\%,66\%, 15\% and 98\%. The 4item bFHQ was more sensitive than eFHQ and took less than 10 minutes for each patient to complete.

Conclusions Patient-administered bFHQ may serve as an adequate screening tool to triage women with OC for further genetic assessment for LS, especially in centers without access to universal tumor testing for IHC for MMR.

\section{EPV150/\#195 DYNAMICS OF THE INCIDENCE RATES FOR GYNECOLOGIC CANCER IN UZBEKISTAN}

S Djanklich*, M Tillyshaykhov, N Zakhirova, A Berkinov. Republican Specialized ScientificPractical Medical Center of Oncology and Radiology, Gynecological, Tashkent, Uzbekistan

\subsection{6/ijgc-2021-IGCS.220}

Objectives Estimate trends of change in cancer morbidity for cervix, uterine corpus, and ovaries of the female population of Uzbekistan over a 10-year period (2010-2020).

Methods We collected cervical, uterine and ovarian cancer incidence data from official statistics in Uzbekistan for the years 2010-2020.

Results For the period 2010-2020, there were 16137 cases of cervical cancer, 5772 cases of uterine cancer and 7562 cases of ovarian cancer for the first time. During the analyzed period, more than $55 \%$ of cervical cancer cases, $70 \%$ of uterine cancer and more than $42 \%$ of ovarian cancer were registered at stages I-II. In 2010 there were 7738 patients with cervical cancer, 5253 patients with uterine cancer and 3503 cases with ovarian cancer, meanwhile, in 2020 there were 9125, 5 017, 4391 cases with cervical, uterine and ovarian cancer accordingly. The maximum incidence rate of gynecologic cancer was observed at the age of 45-65 years. The proportion of stage I cervical cancer cases was highest in Namangan region (30.4\%), of uterine cancer in Tashkent city $(60.2 \%)$ and ovarian cancer in andijan region (19.6\%) compared with other regions.

Conclusions Our results suggest constant increase in incidence rate of cervical, uterine and ovarian cancer in Uzbekistan. For the last 10 years percentage of I-II stages of cervical, uterine and ovarian cancer was not so high. Every year there is a tendency in increasing of patients with gynecologic cancer. But from 2021 to 2025 , it is planned to screen 3,473,902 women for cervical cancer.

\section{EPV151/\#376 GENETIC TESTING REFERRALS FOR ENDOMETRIAL CANCER PATIENTS: CAN WE DO BETTER?}

${ }^{1} \mathrm{M}$ Shah*, ${ }^{2} \mathrm{D}$ Kupperman, ${ }^{3} \mathrm{~S}$ Stroever, ${ }^{3} \mathrm{~L}$ Riddle, ${ }^{3} \mathrm{~A}$ Kliss, ${ }^{4} \mathrm{~L}$ Chuang. ${ }^{1}$ Nuvance Health Network, Internal Medicine, Danbury, USA; ${ }^{2}$ Nuvance Health Network, Genetic Counseling, Danbury, USA; ${ }^{3}$ Nuvance Health, Research and Innovation, Danbury, USA; ${ }^{4}$ Nuvance Health, Obstetrics, Gynecology, Reproductive Biology, Division of Gynecologic Oncology, Danbury, USA

\subsection{6/ijgc-2021-IGCS.221}

Objectives Mismatch repair gene testing for patients with endometrial cancer assists in identifying suspected mutation 
carriers with Lynch syndrome. The purpose of this study is to examine the factors predictive of endometrial cancer patients' adherence to genetic counseling referrals for genetic testing. Methods An IRB-approved retrospective study was conducted on eligible patients identified at multidisciplinary tumor boards between January 2016 to October 2019. Our primary outcome was genetic testing completion when recommended by a genetic counselor. Data collected included age at diagnosis, ethnicity, stage, metastasis, mismatched repair deficiency testing, presence of Lynch syndrome, and genetic counselor presence at the tumor board. We performed univariate analyses to test for group differences using the independent student's ttest for age and Fisher's exact test for categorical variables. We performed multivariable logistic regression to determine the independent odds of genetic testing, including age, metastasis, and stage.

Results Our sample included 165 patients, and genetic testing was recommended for 30 (18.2\%). Sixteen of the $30(53.3 \%)$ patients recommended for testing adhered to the recommendation. As a result, three patients were diagnosed with Lynch syndrome. There was a significant difference in age between those tested versus those who did not get tested. On multivariable analysis, for every one year increase in age, the odds of genetic testing decreased. There was a trend toward reduced odds of genetic testing among patients with stage III/ IV compared to I/II cancer.

Conclusions Our findings suggest an opportunity to increase the genetic testing referral process for older patients and possibly those with more advanced disease.

\section{EPV152/\#655 GENETIC PROFILE BY WHOLE EXAM SEQUENCING OF BORDERLINE OVARIAN TUMORS: SERIES OF 32 PATIENTS}

${ }^{1} \mathrm{D}$ Atallah, ${ }^{*}{ }^{2}$ El El Feghaly, ${ }^{3} \mathrm{E}$ Choueiry, ${ }^{3} \mathrm{~N}$ Jalkh, ${ }^{4} \mathrm{~A}$ Khaddage, ${ }^{4} \mathrm{M}$ Akiki, ${ }^{5} \mathrm{H}$ Kourie, ${ }^{1} \mathrm{~N}$ El Kassis, ${ }^{5} \mathrm{G}$ Chahine, ${ }^{1} \mathrm{M}$ Moubarak. ${ }^{1}$ Hôtel-Dieu de France University Hospital, Gynecologic Oncology, Beirut, Lebanon; ${ }^{2}$ Hôtel-Dieu de France University Hospital, Obstetrics and Gynecology, Beirut, Lebanon; ${ }^{3}$ Université Saint Joseph, Genetics, Beirut, Lebanon; ${ }^{4}$ HôtelDieu de France University Hospital, Pathology, Beirut, Lebanon; ${ }^{5}$ Hôtel-dieu de France University Hospital, Oncology, Beirut, Lebanon

\subsection{6/ijgc-2021-IGCS.222}

Objectives Borderline ovarian tumors are defined as non-invasive epithelial ovarian tumors which can have an intraperitoneal extension. Molecular studies have shown a correlation between the patient's response to chemotherapeutic treatments adjunct to surgery and the tumor's genetic profile, especially related to the KRASand BRAF genes. This study aims to assess the molecular profile of BOTs in the Lebanese population by Whole Exome Sequencing (WES) and correlate the results with patients' clinical profiles.

Methods 33 tumors belonging to 32 Lebanese patients presenting with BOTs, diagnosed at Hôtel Dieu de France were included. A total of 234 genes involved in different germinal and somatic types of cancer were analyzed using Next Generation Sequencing in the 33 included tumors. Genetic variants detected in more than $5 \%$ of the reads, with a sequencing depth $\geq 50 \mathrm{x}$, were selected.
Results Among 33 tumors, 18 were serous, 12 mucinous and 3 seromucinous. Molecular analysis of tumors allowed us to detect mutations in genes involved in the MAP Kinase (MAPK) cascade and in the DNA repair mechanism. Our initial analysis revealed an association between defects in DNA Double-Strand Break repair and occurrence of mucinous BOT, in $75 \%$ of cases. Mutations affecting MAPK signaling pathway were detected in $46.9 \%$ of BOT.

Conclusions Here we report the molecular profile of BOT in the Lebanese population. This is the first study associating the DNA repair pathway to BOT. The inclusion of further patients is essential to validate our hypothesis and to better delineate the mechanisms of the disease, thus allowing the implementation of targeted therapeutic approaches.

\section{EPV153/\#66 RELIABILITY, COSTS AND APPLICABILITY OF THE WHOLE BODY DEXA SCAN IN THE ASSESSMENT OF MUSCLE MASS AFTER RISK-REDUCING SALPINGO-0OPHORECTOMY IN BRCA1/2 PV CARRIERS}

${ }^{1}$ I Stroot*, ${ }^{1} \mathrm{~A}$ Stuursma, ${ }^{2} \mathrm{~K}$ Vermeulen, ${ }^{3} \mathrm{M}$ Greuter, ${ }^{4} \mathrm{R}$ Slart, ${ }^{1} \mathrm{M}$ Mourits, ${ }^{2} \mathrm{~T}$ De Bock. ${ }^{1}$ University Medical Center Groningen, Gynaecology and Obstetrics, Groningen, Netherlands; ${ }^{2}$ University Medical Centre Groningen, Epidemiology, Groningen, Netherlands; ${ }^{3}$ University Medical Center Groningen, Radiology, Groningen, Netherlands; ${ }^{4}$ University Medical Center Groningen, Nuclear Medicine, Groningen, Netherlands

\subsection{6/ijgc-2021-IGCS.223}

Objectives Sarcopenia is the quantitative and qualitative loss of skeletal muscle, which may be associated with acute surgical menopause after risk-reducing salpingo-oophorectomy (RRSO) in BRCA1/2 PV carriers. Magnetic Resonance Imaging (MRI) and Computed-Tomography (CT) are currently the golden standard to measure muscle mass. Dual Energy X-ray Absorptiometry (DEXA) is less costly with less radiation exposure. As there are no data on its intra- and inter-observer variability, the aim of this study was to establish if the DEXA scan could be a reliable alternative to CT or MRI in the analysis of muscle mass.

Methods To assess inter- and intra-observer variability, DEXA scans of the lower extremities of women 10 or more years after RRSO were analyzed by two observers, who independently analyzed each scan twice. Information about costs and radiation dose from the DEXA, CT and MRI were collected from literature.

Results DEXA scans of 34 women with a median age of 58.0 years (range 45.0-73.0) and a median BMI of 24.6 (range 18.0-47.2) were analysed. Inter-observer variability had an Interclass correlation coefficient (ICC) of 0.997 and acceptable limits of agreement. Intra-observer variability was also low: $\mathrm{ICC}_{\text {observer } 1}: 0.998$ and $\mathrm{ICC}_{\text {observer } 2}: 0.997$. Observer 1 had lower limits of agreement. Costs and radiation exposure were lower for DEXA than CT and MRI.

Conclusions The assessment of muscle mass of the lower extremities with DEXA scan has a high reliability, is less costly and has a lower radiation dose than CT and MRI. DEXA scan may be a good alternative for measuring muscle mass to diagnose sarcopenia. 